Computing and Informatics, Vol. 40, 2021, 833-849, doi: $10.31577 /$ cai_2021_4_833

\title{
TRIGGER PERFORMANCE MONITORING AND RATE PREDICTIONS PREPARATION FOR RUN 3 AT ATLAS EXPERIMENT
}

\author{
Aleksandra POREBA* \\ CERN \\ 211 Geneva 23 \\ Switzerland \\ e-mail: aleksandra.poreba@cern.ch
}

\begin{abstract}
Bespoke Cost Monitoring software collates data on the performance of all aspects of the ATLAS experiment's High Level Trigger software. These data are exported for subsequent analysis offline, and are used to understand the resource usage of the individual trigger selections in terms of the amount of CPU time and the amount of raw detector data which was required to perform the selection. For the LHC's Run 3, the ATLAS High Level Trigger is re-implemented in a multi-threaded framework with both intra-event and inter-event algorithm parallelism. We will describe some of the complications and considerations which arise from monitoring event metrics in a highly parallel environment.
\end{abstract}

Keywords: CERN, ATLAS, trigger, cost monitoring

\section{INTRODUCTION}

The ATLAS experiment [1] at the LHC is a multipurpose particle detector designed to record physics events for a wide range of measurements and searches. It consists of inner and muon tracking detectors, electromagnetic and hadronic calorimeters, and uses magnetic fields produced by solenoid and toroid magnets. The detector records the signals produced by the collisions delivered by the LHC at a rate of 40 $\mathrm{MHz}$ (for proton-proton data taking). Physics data is collected during time periods called Runs. Between them, upgrade break takes place called "Long Shutdown". Two

* Copyright 2021 CERN for the benefit of the ATLAS Collaboration. CC-BY-4.0 license 
Runs already took place: Run 1 (2009-2013) and Run 2 (2015-2018), the Run 3 is expected to start in 2022 and last until 2025.

The ATLAS experiment was not designed to record all of collisions results, as most of the recorded data is not interesting for physics analysis. The ATLAS Trigger system (architecture is presented in the Figure 1) aims to select the data created by interesting collisions in real time. The first-level trigger is implemented in hardware and uses a subset of the detector information to accept events at a rate below $100 \mathrm{kHz}$ during Run 2. The L1 rate is limited by the hardware, hence it is expected to stay the same for Run 3. The Level-1 trigger is followed by a software-based trigger, High Level Trigger (HLT), with target average rate $1.5 \mathrm{kHz}$ for Run 3, depending on the data-taking conditions. An extensive software suite [2] is used for real and simulated data reconstruction and analysis, for operation and in the trigger and data acquisition systems of the experiment.

The HLT runs on a large computing farm of CPUs. During the shutdown between data-taking periods, the machines are being upgraded in order to achieve higher processing efficiency. However, estimations of HLT resources usage are also required to inspect if the new software will be able to process the data with a desired frequency. The Trigger system is equipped with such tools to predict the software performance in advance of collisions.

\subsection{Trigger Menu}

The HLT filtering process consists of selection steps, referred to as chains. Each chain has defined physics thresholds that are required to consider an event as interesting for physics analysis. The chains are defined in the Trigger Menu, different menus are prepared for different data taking types (heavy ion, proton-proton physics).

In each step, a series of physics reconstruction algorithms are executed in order to retrieve the details of the recorded event. Afterwards, the chains are tested to check if the given criteria of the chain were fulfilled. If not, further algorithms will not be executed and the event is rejected. The steps of the chain are processed until either the chain rejects the event at the end of a step, or passes the final step and hence passes the event.

The order of algorithms within a chain relies on an early-rejection mechanism. The early steps should be the most selective and rejective. If a step fails, steps that depend on it are removed and, as a consequence, many chains will be rejected. This way the computing resources usage can be reduced.

\subsection{Cost Monitoring}

During online data taking or data reprocessing monitoring data is collected in addition to physics data. One of the available monitors is the Cost Monitor that enables data-driven CPU usage predictions. The "cost" stands for the execution time of algorithms or events, based on which the resources estimations can be performed 


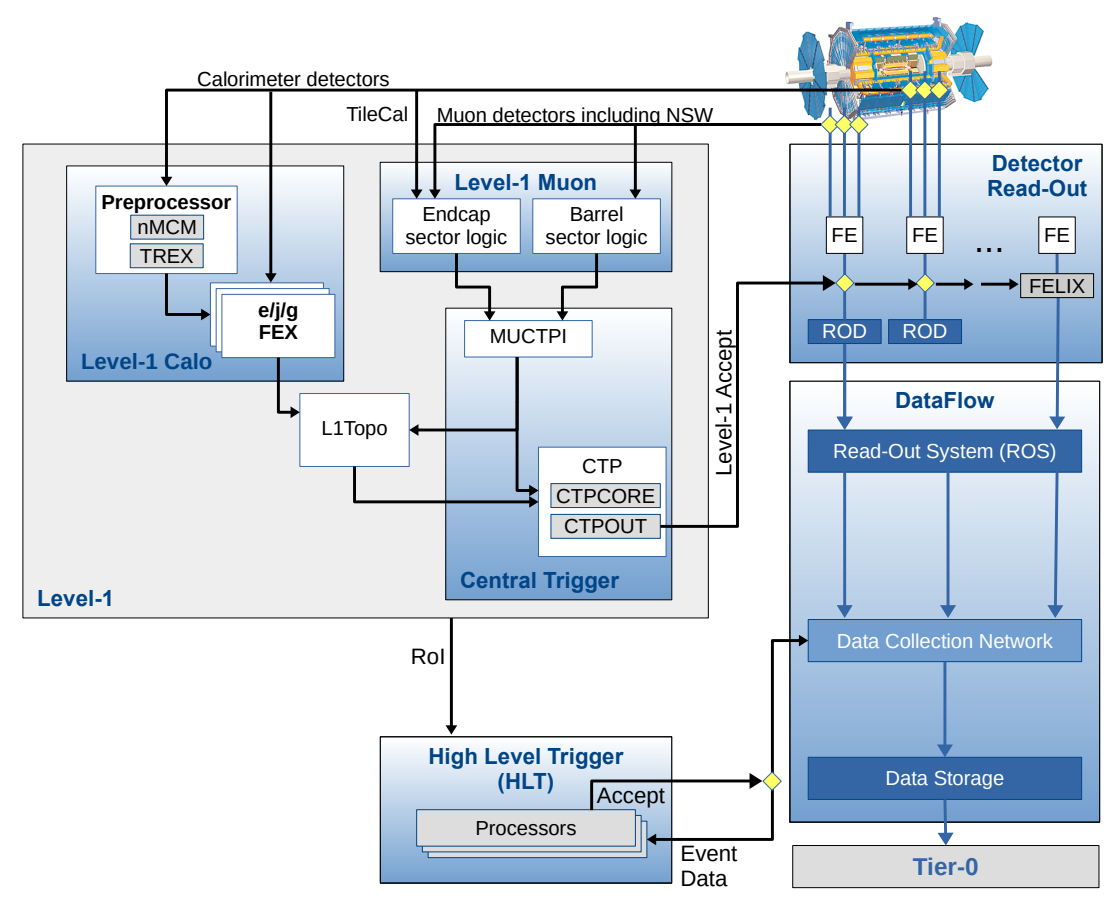

Figure 1. Functional diagram of the ATLAS Trigger and Data Acquisition system in Run 3 [3]. Details of the components can be found in [4].

in advance of collisions. In the Trigger development phase, it can be used to spot problematic resource usage and optimize the HLT software.

When collecting the data, $10 \%$ of all the events are monitored by the Cost Monitor. With the HLT execution reaching up to $100 \mathrm{kHz}$ rate, up to $10 \mathrm{kHz}$ of monitoring data is recorded. Statistics relating to the execution of the algorithm and its request for raw detector data are saved to perform the offline analysis: start and stop timestamps, requested data size, and status flags.

The Cost processing is based on a separate HLT output data stream containing recorded Cost Monitoring details. After processing the monitoring data a ROOT [5] file is produced with a set of histograms illustrating the execution performance of different Trigger areas, for example, algorithms, chains, threads occupancy, or the read out system. A set of Comma Separated Value files is created from the monitoring histograms with different available summaries presenting alternate overviews of the resource usage. The result can be displayed on the TriggerCostBrowser website, equipped with tools to enable easy browsing of the summary tables. The process and result structure was described in detail in [6]. 


\subsection{Multithreaded Framework}

The HLT software is based on top of the Athena [7] framework, which is also used in the ATLAS Collaboration to perform physics simulation, reconstruction and data analysis. It is based on Gaudi [8], inter-experiment framework providing the foundations for High Energy Physics data processing applications.

Both Athena and Gaudi were designed for single threaded operations. Since that time the computing market has changed to favoring multi-core processors for higher processing efficiency. The same is not true for memory; the prices are oscillating over the last years and no longer matching the decreasing trend. The observed trends are presented in Figures $2 \mathrm{a}$ ) and $2 \mathrm{~b}$ ). These changes encourage a concurrent framework approach with shared memory rather than single threaded processing.

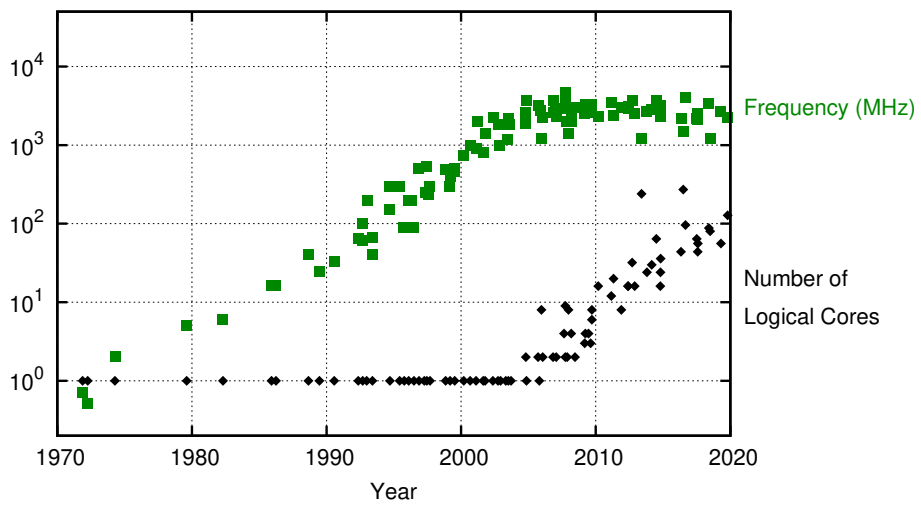

a)

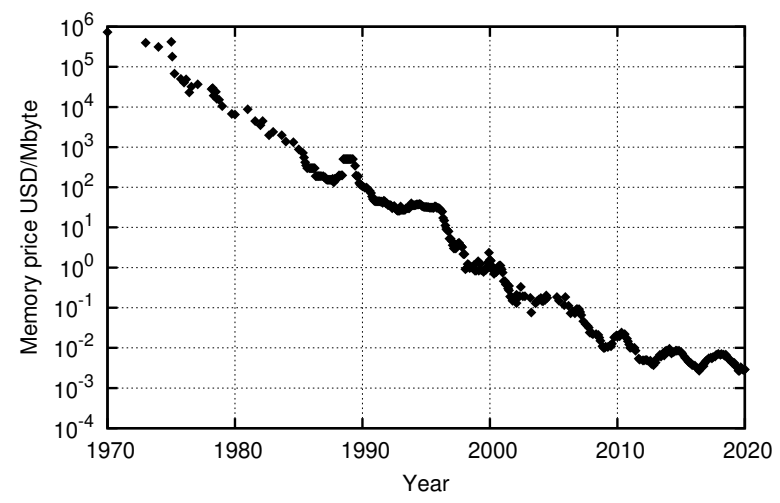

b)

Figure 2. a) CPU frequency and logical core count trends are presented based on data 9]. b) illustrates memory price trends between 1975 and 2019, based on data [10]. 
During Run 1 and 2 chains and their algorithms were executed linearly using a single-threaded approach. The results of algorithm executions were cached and could be reused in the future if required by other chains to minimize the computing costs.

For Run 2 the way of executing HLT software was upgraded to support a multiprocessing approach with Athena instances being forked after initialization. The processes shared the memory at the OS level as long as the pages stayed the same. In case of any change in execution between forks, each one received its own copy of that page. The memory management of Athena in Run 2 was described in [11].

For Run 3 both Athena and Gaudi are redesigned to support both the multiprocess and multi-threaded mode. The new version of Athena, AthenaMT [12] is based on Gaudi Hive [13] with a concurrent task scheduler based on Intel Thread Building Blocks (TBB) [14].

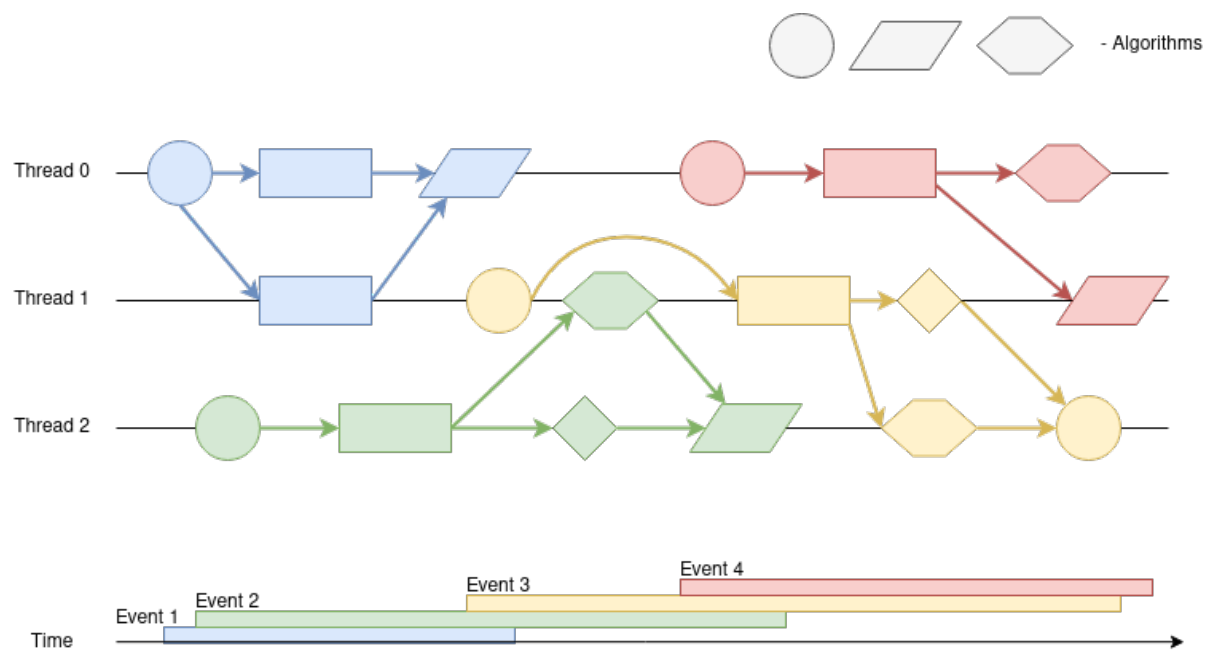

Figure 3. Schematic flowchart describing concurrent event processing in AthenaMT. Each shape corresponds to a type of algorithm and each colour corresponds to data associated with one event. Data flow is represented by arrows.

The High Level Trigger was re-implemented in the new concurrent Athena framework. Instead of processing a single chain at a time then caching a result, a step is now executed only once, for all of the chains with the same input. The processing is parallel both inter-event (multiple events can be processed in parallel), intra-event (algorithms for one event can be executed in parallel provided they have needed input), and also in-algorithm (algorithms can use multithreading specific operations, for example concurrent loops). A schematic example of concurrent events processing in HLT is shown in Figure 3. 


\section{COST MONITORING IN ATHENAMT}

The multi-threading redesign changes also affected the Cost Monitoring. An algorithm execution can no longer be associated with only one chain, which required revising the way the Cost data is created.

Which chains actually executed the algorithm (i.e. chains which were not already rejected in an earlier step) can be determined based on the navigation data generated during the execution of the HLT. After each algorithm execution the summary with a result is created and, in the end, saved in the output data stream file for further physics analysis. Based on these results the Cost "Chain Summary" is created by checking if a navigation item of algorithms of the chain was created for the processed event.

In AthenaMT the execution time is the same for all chains that include a given algorithm. In the past if the algorithm result was cached, the second execution time was 0 - cached result was used. This created an illusion of "free" chains, that used only cached results when in fact the cost was spread between other chains that were executed earlier. The new approach is more reliable showing the true cost of the chain.

Table 1 presents a comparison of Run 2 and Run 3 Cost Monitoring results for two chains: selections $\mathrm{A}+\mathrm{B}$ and $\mathrm{A}+\mathrm{C}$ in the order of execution. In the Run 2 Cost Summary the execution time of the $\mathrm{A}+\mathrm{C}$ chain (executed later than the $\mathrm{A}+\mathrm{B}$ chain) is only $50 \mathrm{~ms}$ - cached A result from the first executed chain was used and only $\mathrm{C}$ selection result had to be obtained. In AthenaMT the real execution time of a chain is shown. In the $\mathrm{A}+\mathrm{C}$ chain the $\mathrm{A}$ execution is no longer hidden as a result of caching and the chain execution time does not depend on the order of the chains.

If the order was switched, the results for Run 2 would be different: $250 \mathrm{~ms}$ for $\mathrm{A}+\mathrm{C}$ chain and $100 \mathrm{~ms}$ for the $\mathrm{A}+\mathrm{B}$ chain. The new Cost Monitoring framework is resistant to such changes.

\begin{tabular}{|l|r|}
\hline Algorithm & Execution Time [ms] \\
\hline Selection A & 200 \\
Selection B & 100 \\
Selection C & 50 \\
\hline
\end{tabular}

\begin{tabular}{|l|r|r|}
\hline Chain & $\begin{array}{r}\text { Execution Time Per Event } \\
\text { in Run } 2[\mathrm{~ms}]\end{array}$ & $\begin{array}{r}\text { Execution Time Per Event } \\
\text { in Run } 3[\mathrm{~ms}]\end{array}$ \\
\hline Selections A + B & 300 & 300 \\
Selections A + C & 50 & 250 \\
\hline Total measured time & 350 & 350 \\
\hline
\end{tabular}

Table 1. Representative numbers of mean execution time of two chains containing selections: $\mathrm{A}+\mathrm{B}$ and $\mathrm{A}+\mathrm{C}$ recorded by the Cost Monitoring in Run 2 and Run 3 HLT framework. 
On the other hand, with the new way of measuring execution time of chains, the sum can be higher than the time of execution of the event. Knowing that two chains can execute one algorithm, the sum of all the execution times of the chains will not be equal to the total time of execution of the algorithms. The new changes make time of the chain more accurate when comparing to each other. For a global HLT Cost analysis, the time and number of calls for each algorithm are the most important.

\subsection{Saving Cost Monitoring Data}

The new AthenaMT framework defines a number of processing slots. Incoming events are assigned to an available slot and they will occupy it until the processing of the event is finished. Each slot has its own data store, where the details of the monitored event are saved. Cost Monitoring data from all of the slots is saved to the same output data stream file.

However, during online data taking and reprocessing, additional details can be saved to the Master Slot (set in configuration, by default Slot 0). When the event processed by this slot is monitored by Cost Monitoring, data about events from other slots is collected also into the data store of the Master Slot. The data saving mechanism is illustrated on Figure 4 .

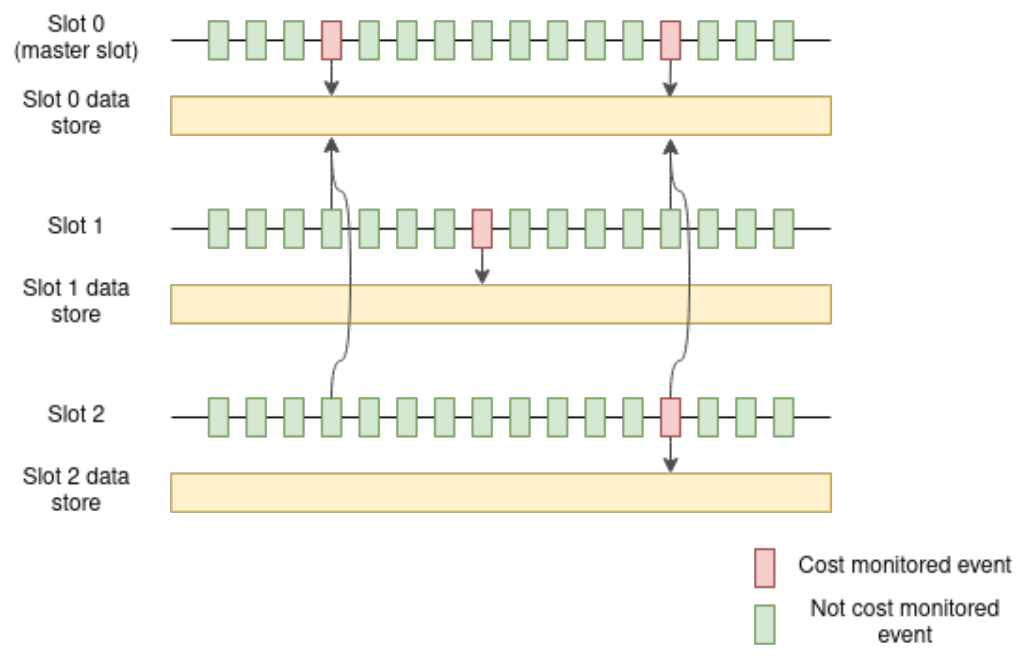

Figure 4. Diagram illustrating Cost Monitoring data saving mechanism with additional data saved to the master slot. Three cases are possible (presented on the diagram from left to right): master slot is monitored, non-master slot is monitored or both master and non-master slots are monitored.

This mechanism is used for thread monitoring in order to retrieve details of what is happening in other slots during current event processing. Events are processed 
at the same time on different slots (mentioned in the Section 1.3 intra event parallelism). With the full information we are able to compare the threading performance of the HLT process over all slots and assigned threads.

The information about the source slot is also saved to the output data stream, necessary for the Cost Processing. Events originated in non-Master Slots that are saved in Master Slot's data store should be ignored for other monitors, for example for algorithms and chains. Otherwise, the same data would be processed twice (the third case on the Figure 4) and the monitored event rate would be higher than 1 in 10. In order to avoid the data bias, the thread monitoring uses only the Master Slot saved data.

\subsection{Thread Monitoring}

Thread monitoring is a new feature possible inside a multi-threaded HLT implementation. Its purpose is to give an overview of the multi-threading efficiency for given forks, event slots and threads configuration. The event execution time is not only determined by all the algorithms execution duration but also by "framework time". It is defined as the time a thread spent outside of scheduled algorithms while waiting for an algorithm to be dispatched.

By looking at how much time it took to process an event (from the earliest algorithm executed on any of the threads for this event to the latest) and the sum of all the execution times of algorithms on each individual thread, the time spent on executing algorithms and on framework related operations can be determined. Algorithms from other slots whose execution spans the global start and stop of the master thread add a degree of uncertainty to this measurement, this component is computed separately as unmonitored time. The three values: algorithm, framework and unmonitored time are illustrated on Figure 5. The way they are computed is described by Equations (1), (2) and (3).

$$
\begin{aligned}
\text { eventTime } & =\text { globalStart }- \text { globalEnd } \\
\text { unmonitoredTime } & =(\text { globalStart }- \text { threadStart })+(\text { threadEnd }- \text { globalEnd }),(2) \\
\text { frameworkTime } & =\text { eventTime }- \text { unmonitoredTime }- \text { algorithmTime }
\end{aligned}
$$

Figures 6 and 7 show the fraction of framework and algorithm time. It is observed that in long events, algorithm time dominates whereas in short events the fraction is closer to being equal, testifying to the good performance of the early rejection system. The framework time is consistent in all events. Different time of processing an event depends on the actual event details. The algorithms are only executed when the conditions are fulfilled and the chain was not rejected earlier. The mean framework time should stay the same for a given configuration, only the algorithm time varies. Analysing the distribution of algorithm time per event and framework time per event, the best configuration can be identified. 


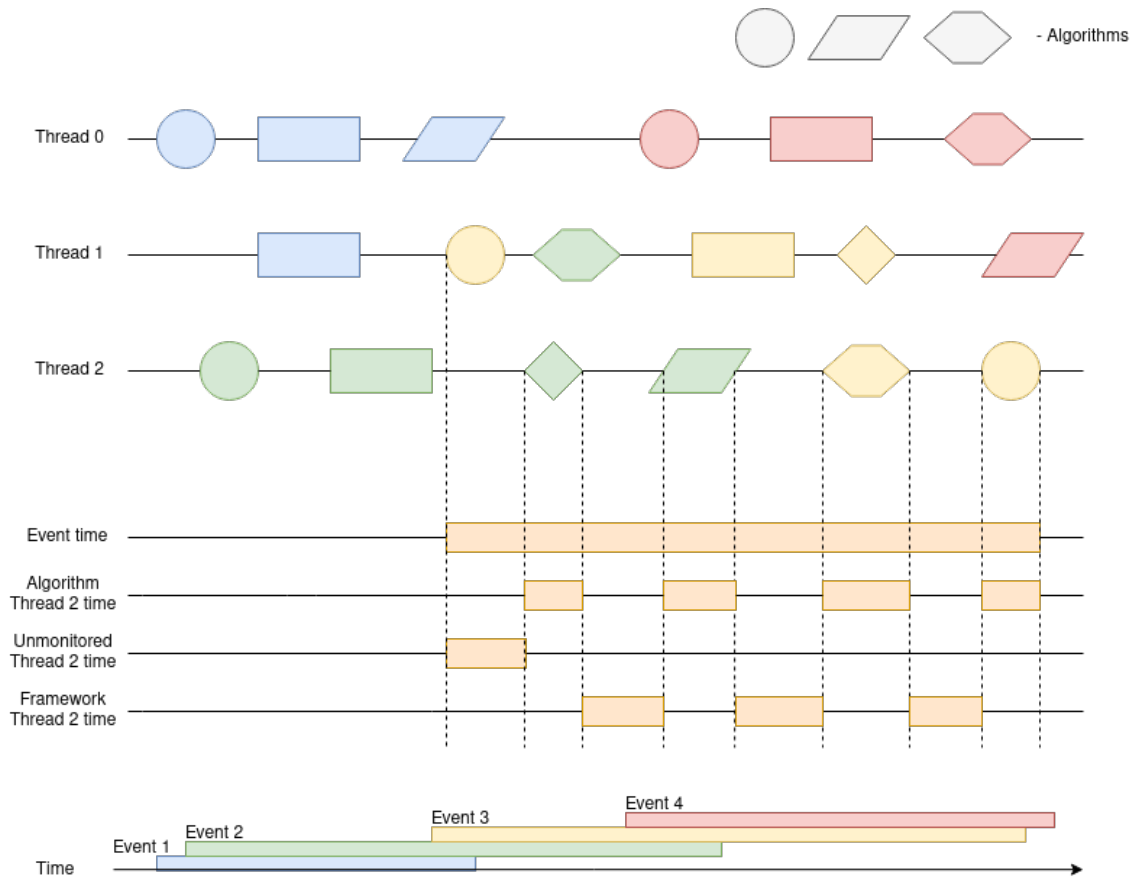

Figure 5. Graphic interpretation of event, unmonitored and framework time for Thread 2 based on the flowchart in Figure 3. During the time of the event window all algorithms are monitored (not only algorithms that are running for the given event).

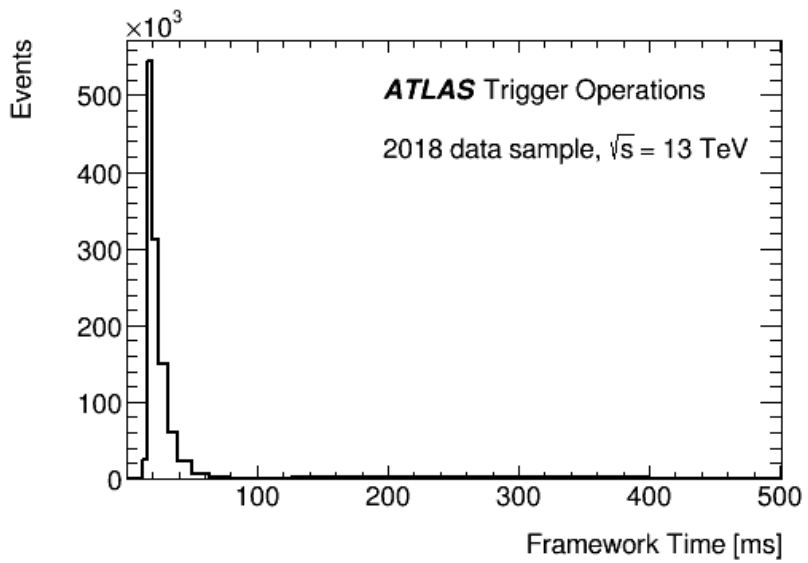

a) 


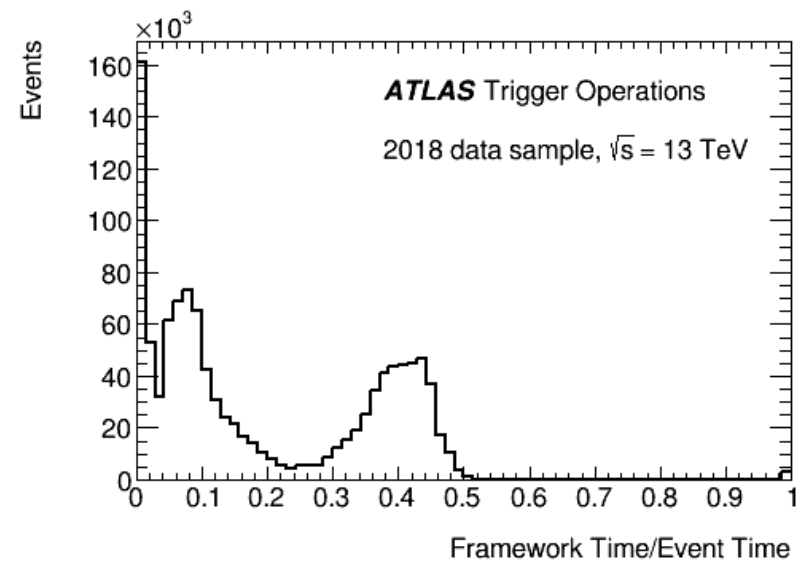

b)

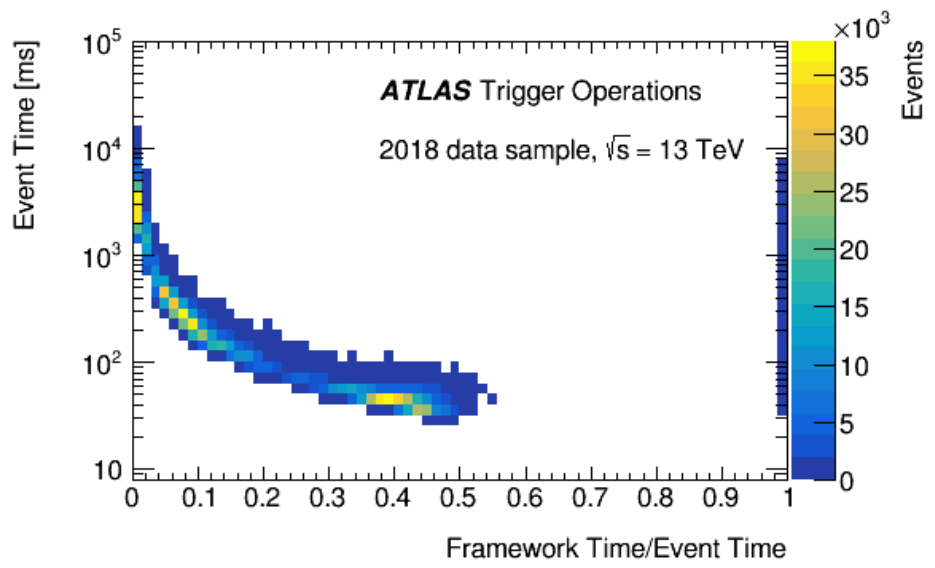

c)

Figure 6. a) Measured framework time for sample of events. b) Example of measured framework time on one thread as fraction of the monitored event time window. c) The fractional time showed as a function of the monitored event time window. Three peaks can be observed on the plots 6 b) and 6c) - one when thread performs framework operations $40 \%$ of the time during short events processing (approximately $100 \mathrm{~ms}$ ). The other two correspond to long events (approximately $300 \mathrm{~ms}$ and $3000 \mathrm{~ms}$ ) when the framework time takes a minor fraction of the event time window. For some of the events included in the data sample the framework time takes $100 \%$, what is happening when the event does not fulfill requirements to trigger any of the algorithm executions. Based on the plot 6 a) it can be observed that for all events the framework time is stable and its mean value equals to $20 \mathrm{~ms}$, despite fluctuations in framework fraction time. The plots were created with a small 2018 data sample that was preloaded into the HLT farm and processed repeatedly. [15] 


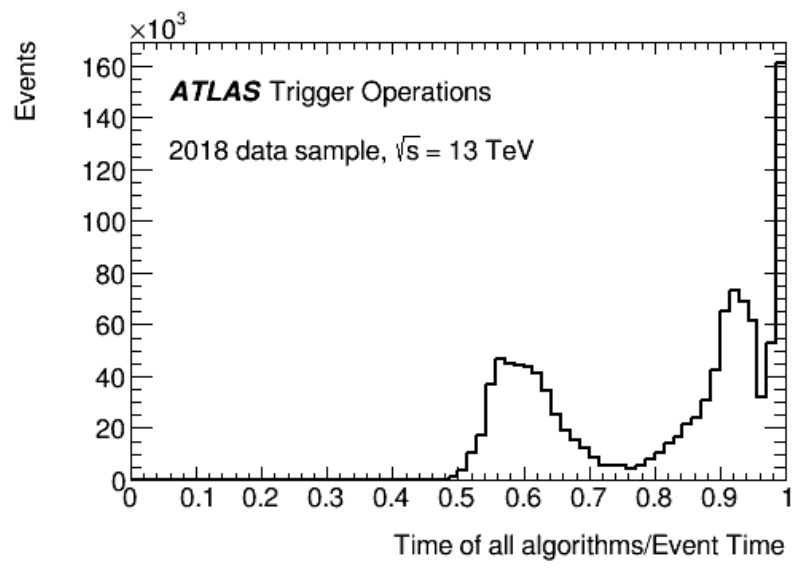

a)

\section{INTERPRETING THE COST RESULTS}

Cost Monitoring was written into the Trigger infrastructure in order to perform detailed offline studies of the system's performance. Based on Cost data collected during the data taking, one can not only estimate CPU resources but also access the details of the HLT execution. The Cost summaries cover executed algorithms, chains, sequences (groups of algorithms), data requests and the aforementioned thread occupancies. One additional summary covers the global parameters of the HLT execution, including mean processing time of the event, number of active events within a given period of time, or number of algorithm calls in the event.

In the following paragraphs the contents of some of the summaries will be discussed. Starting with the "Algorithm Summary", different algorithms and their parameters can be analysed, including:

- Number of calls per event.

- Mean time of execution per event.

- Total algorithm execution time and what fraction of total HLT walltime it is.

- How often the algorithm was called and how often algorithm was executed in an event.

- If the algorithm requested any data and if these data were cached locally or fetched over the network.

An example of "Algorithm Summary" table is presented in Figure 8

The expensive algorithms can be identified by a long algorithm total time. Not only the measured time is important but also what percentage of the total time the execution took. 


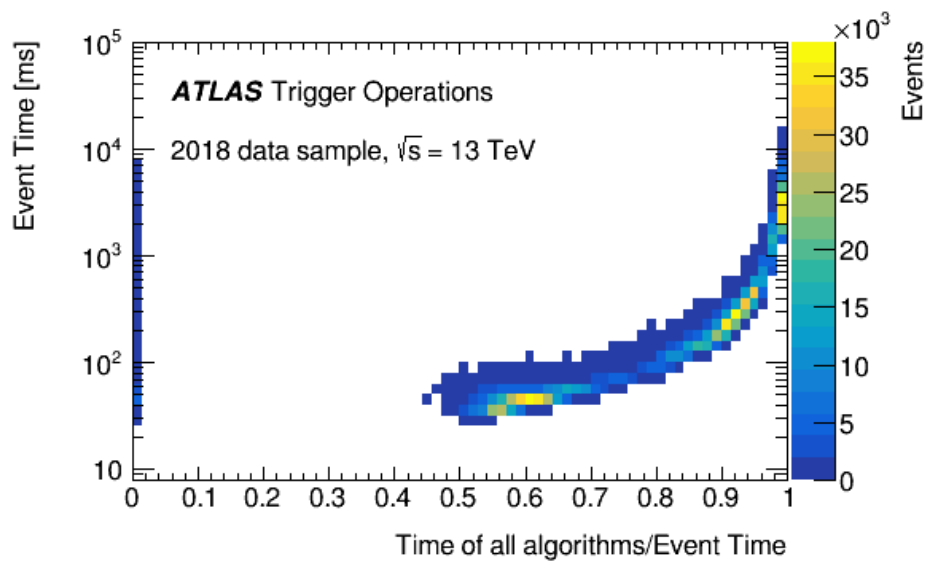

b)

Figure 7. a) Example of measured time of all the algorithms executed on the thread as fraction of the monitored event time window. b) The fractional time showed as a function of the monitored event time window. Three peaks can be observed in the plots - one when thread processes algorithms $60 \%$ of total time, which happens for short events (approximately $100 \mathrm{~ms}$ ), when the event data does not fulfill the requirements to trigger execution of time consuming algorithms. The other two peaks represent long events (approximately $300 \mathrm{~ms}$ and $3000 \mathrm{~ms}$ ) when algorithm processing takes majority of the total time. The peaks correlate with recorded times of algorithm execution per event. For some of the events included in the data sample the algorithm processing takes $0 \%$ of the time, which is happening when the event does not fulfill requirements to trigger any of the algorithm executions. The rest of the time is spent for so-called "framework time". The plots were created with a small 2018 data sample that was preloaded into the HLT farm and processed repeatedly. [15]

The reasons for poor algorithm performance could be multiple algorithm calls per event or the time spent waiting for data caused by a high rate of data requests from the read-out system. The number of chains that executed the algorithm should be also checked. A chain-algorithm mapping available on the website can be used to directly access associated chains' summaries. Naturally, some of the algorithms will be more expensive than the others, the computational cost of each selection must be balanced against the available resources and the physics goals of the ATLAS collaboration.

Another important summary to take into the account performing Cost analysis is the "Chain Summary" containing details about execution of chains, including:

- Number of events in which chain was active.

- Execution rate of chain. 


\begin{tabular}{|c|c|c|c|c|c|c|c|c|c|}
\hline \multicolumn{7}{|c|}{ Algorithm Summary } & \multicolumn{3}{|c|}{ ATLAS Trigger Operation * } \\
\hline 50 & $\mathbb{1} 4$ & Page 1 & of 55 & $v \|$ & Displaying $1 \mathrm{t}$ & to 50 of 2731 items & & & \\
\hline \multicolumn{4}{|l|}{ Find } & Name $v$ & \multicolumn{5}{|c|}{ Reprocessing of 2018 data $13 \mathrm{TeV}$} \\
\hline Name & Active Events & Calls/Event & Calls $>1000 \mathrm{~ms}$ & Event Rate $[\mathrm{Hz}]$ & Call Rate $[\mathrm{Hz}]$ & Alg Total Time $[\mathrm{s}]$ & Alg Total Time [\%] & Alg Total Time/Call [ms] & Alg Total Time/Event [ms] \\
\hline Algorithm 01 & $2.32 E+07$ & 4.295 & 32.77 & $6.90 \mathrm{E}+03$ & $3.13 E+04$ & $2.96 E+05$ & 0.1319 & 2.968 & 12.75 \\
\hline Algorithm 02 & $2.32 E+07$ & 4.295 & 597.3 & $6.90 \mathrm{E}+03$ & $3.13 E+04$ & $1.01 E+06$ & 0.4485 & 10.68 & 43.42 \\
\hline Algorithm 03 & $2.32 \mathrm{E}+07$ & 4.295 & 0 & $6.90 E+03$ & $3.13 E+04$ & $1.60 \mathrm{E}+04$ & 0.007122 & 0.1773 & 0.7215 \\
\hline Algorithm 04 & $2.32 E+07$ & 4.295 & 0 & $6.90 E+03$ & 3.13E+04 & $1.73 E+04$ & 0.007717 & 0.19 & 0.7778 \\
\hline Algorithm 05 & $2.32 E+07$ & 4.295 & 0 & $6.90 \mathrm{E}+03$ & $3.13 E+04$ & $5.23 E+04$ & 0.02327 & 0.5436 & 2.259 \\
\hline Algorithm 06 & $3.28 E+06$ & 1 & 0 & 976.3 & 4.39E+03 & $1.78 E+03$ & 0.0007913 & 0.541 & 0.541 \\
\hline Algorithm 07 & $3.28 E+06$ & 1 & 0 & 975.1 & $4.39 \mathrm{E}+03$ & $2.31 E+03$ & 0.001026 & 0.7015 & 0.7015 \\
\hline Algorithm 08 & $7.38 E+06$ & 1 & 0 & $2.20 \mathrm{E}+03$ & $9.88 E+03$ & $2.44 E+03$ & 0.001085 & 0.3298 & 0.3298 \\
\hline Algorithm 09 & $2.32 E+07$ & 4.295 & $5.28 \mathrm{E}+03$ & $6.90 E+03$ & $3.13 E+04$ & $2.09 \mathrm{E}+05$ & 0.09307 & 2.22 & 9.033 \\
\hline Algorithm 10 & $2.32 E+07$ & 4.295 & 997.9 & $6.90 \mathrm{E}+03$ & 3.13E+04 & $3.19 \mathrm{E}+06$ & 1.42 & 31.93 & 137.1 \\
\hline Algorithm 11 & $2.32 E+07$ & 4.295 & 0 & $6.90 E+03$ & $3.13 E+04$ & 357.9 & 0.0001593 & 0.4817 & 0.1967 \\
\hline Algorithm 12 & $2.32 E+07$ & 4.295 & 0 & $6.90 E+03$ & $3.13 E+04$ & $2.50 E+04$ & 0.01114 & 0.271 & 1.094 \\
\hline Algorithm 13 & $2.32 E+07$ & 4.295 & 0 & $6.90 \mathrm{E}+03$ & 3.13E+04 & $4.36 \mathrm{E}+03$ & 0.001941 & 0.2222 & 0.3918 \\
\hline Algorithm 14 & $2.32 E+07$ & 4.295 & 498.9 & $6.90 E+03$ & $3.13 E+04$ & $1.64 E+06$ & 0.7288 & 17.76 & 70.59 \\
\hline Algorithm 15 & $1.59 \mathrm{E}+07$ & 1 & 531.7 & $4.74 \mathrm{E}+03$ & $2.13 E+04$ & $3.65 E+03$ & 0.001624 & 0.2961 & 0.2961 \\
\hline
\end{tabular}

Figure 8. Representation of Algorithm Summary table available on TriggerCostBrowser website containing details about the algorithm executions. They include number of events in which algorithm was activated, number of algorithm calls per event, rate of algorithm calls, rate of events in which algorithm was executed, algorithm call duration or total time of algorithm execution. Created based on reprocessing 2018 proton-proton collision data with the latest HLT software. [15]

- Mean time spent for this chain per event.

- Total chain execution time and what fraction of total HLT walltime it is.

- How many algorithms were called by chain on average.

The table also shows information about group of the chain, which tells the signature the chain belongs to. Using that feature signature experts can analyze the cost of chosen groups. An example of "Chain Summary" table is presented in the Figure 9

The Chain Summary is created using collected details about the execution of the algorithms. In the concurrent HLT framework, chains executions overlap and they share the same algorithms. Using chain-algorithm mapping presented in Figure 10 related algorithms can be explored and the most expensive ones identified.

Lastly, many studies are performed with different threading configurations. Based on the Cost metadata of the test, displayed on the website, the experts can analyse performance of the HLT with different forks, threads and slots combinations. The saved information include algorithm, framework and unmonitored time. In the future, also additional details about the machines HLT was run on will be saved to the metadata. The tested combinations include pure multi-processing (many forks, one thread and slot), pure multi-threading (one fork, many threads and slots) and 


\begin{tabular}{|c|c|c|c|c|c|c|c|c|c|}
\hline \multicolumn{6}{|c|}{ Chain Summary } & \multicolumn{4}{|c|}{ ATLAS Trigger Operations } \\
\hline a. 50 & IS \& Page 1 & of & $1 \| B$ & Displaying 1 to 500 & of 274 items & Reproces & ssing of 20 & 18 data 13 & $\mathrm{TeV}$ \\
\hline Name & Group & Active Events & Time Per Event [ms] & Execute Rate $[\mathrm{Hz}]$ & Pass Fraction [\%] & Calls $>1000 \mathrm{~ms}$ & Total Chain Time [s] & Total Chain Time [\%] & Run Algs/Event \\
\hline Chain 01 & GroupB, GroupH, GroupM, & $2.19 \mathrm{E}+08$ & 0.4298 & $6.51 E+04$ & 100 & 0 & $6.13 E+04$ & 0.0268 & 4 \\
\hline Chain 02 & GroupA, GroupD, GroupE, Gro & $5.73 E+06$ & 8.859 & $1.70 \mathrm{E}+03$ & 0 & 498.9 & 4.99E+04 & 0.02179 & 12.93 \\
\hline Chain 03 & GroupB, Groupl, GroupM, & $2.19 E+08$ & 0 & $6.51 E+04$ & 100 & 0 & 0 & 0 & 0 \\
\hline Chain 04 & GroupA, GroupJ, GroupN. & $1.30 \mathrm{E}+07$ & 178.3 & $3.86 \mathrm{E}+03$ & 0.9728 & $1.89 \mathrm{E}+03$ & $2.31 E+06$ & 1.009 & 58.71 \\
\hline Chain 05 & GroupA, GroupC, GroupG, & $1.30 \mathrm{E}+07$ & 393.3 & $3.86 \mathrm{E}+03$ & 1.188 & $1.09 E+06$ & $5.11 E+06$ & 2.233 & 14.63 \\
\hline Chain 06 & GroupA, Groupc, GroupG, & 1.30E+07 & 337.5 & $3.86 \mathrm{E}+03$ & 1.292 & $1.07 E+06$ & 4.39E+06 & 1.917 & 11.16 \\
\hline Chain 07 & GroupA, GroupC, GroupG, & $1.30 \mathrm{E}+07$ & 199.1 & $3.86 \mathrm{E}+03$ & 1.415 & $3.35 E+05$ & $2.59 E+06$ & 1.13 & 11.96 \\
\hline Chain 08 & GroupA, Groupk, GroupO & 1.30E+07 & 132.1 & $3.86 E+03$ & 0.7324 & 32.77 & $1.72 E+06$ & 0.7497 & 10 \\
\hline Chain 09 & GroupA, GroupJ, GroupN, & $1.30 \mathrm{E}+07$ & 171.5 & $3.86 E+03$ & 0.9564 & $1.89 \mathrm{E}+03$ & $2.22 E+06$ & 0.9707 & 56.39 \\
\hline Chain 10 & GroupA, Groupc, GroupG, & $1.30 \mathrm{E}+07$ & 391.4 & $3.86 E+03$ & 1.468 & 1.10E+06 & $5.09 E+06$ & 2.222 & 14.63 \\
\hline Chain 11 & Groupc, GroupG, GroupA, & $1.30 \mathrm{E}+07$ & $1.81 E+03$ & $3.86 E+03$ & 0.03779 & 8.17E+06 & $2.35 E+07$ & 10.26 & 23.86 \\
\hline Chain 12 & GroupA, GroupJ, GroupN, & $1.30 \mathrm{E}+07$ & 237.6 & $3.86 E+03$ & 0.406 & $2.79 E+05$ & $3.08 E+06$ & 1.346 & 57.23 \\
\hline Chain 13 & GroupA, GroupC, GroupG, & $1.30 \mathrm{E}+07$ & 373.3 & $3.86 \mathrm{E}+03$ & 1.325 & $1.07 E+06$ & 4.86E+06 & 2.121 & 11.54 \\
\hline Chain 14 & Groupc, GroupG, GroupA, & 1.30E+07 & $1.81 E+03$ & $3.86 \mathrm{E}+03$ & 0.02859 & $8.17 E+06$ & $2.35 E+07$ & 10.26 & 23.86 \\
\hline Chain 15 & GroupA, GroupJ, GroupN, & $1.30 \mathrm{E}+07$ & 244.7 & $3.86 \mathrm{E}+03$ & 0.4037 & $2.81 E+05$ & $3.17 E+06$ & 1.386 & 59.56 \\
\hline
\end{tabular}

Figure 9. Representation of Chain Summary table available on TriggerCostBrowser website containing details about the chain executions. They include groups the chain belongs to, number of events in which chain was activated, chain execution rate, number of algorithm calls that chain made and chain duration. Created based on reprocessing 2018 proton-proton collision data with the latest HLT software. [15]

\begin{tabular}{|cr|}
\hline Chain Algorithms & ATLAS Trigger Operation \\
\hline 0. RoRSeqFilter [AllChains calls:2.19E+08] & Reprocessing of \\
\hline 1. InputMakerForRol [AllChains calls:7.94E+07] & 2018 data 13 TeV \\
\hline 2. HLTCaloCellMaker [AllChains calls:7.94E+07] & \\
\hline 3. TrigCaloClusterMaker [AllChains calls:7.94E+07] \\
\hline 4. PseudoJetAlgorithm [AllChains calls:6.19E+07] \\
\hline 5. JetRecAlg [AllChains calls:6.13E+07] \\
\hline 6. EventDensityAthAlg [AllChains calls:6.19E+07] \\
\hline 7. JetRecAlg [AllChains calls:6.13E+07] \\
\hline 8. JetViewAlg [AllChains calls:6.13E+07] \\
\hline 9. TrigJetHypoAlg [AllChains calls:6.13E+07] \\
\hline 10. PassthroughComboHypo [AllChains calls:3.94E+07] \\
\hline
\end{tabular}

Figure 10. Representation of Chain Item Summary on TriggerCostBrowser website that lists all algorithms related to a particular chain. In this example a jet reconstruction chain is presented. Each algorithm displays its class and the number of calls that it made ("AllChains calls"). Created based on reprocessing 2018 proton-proton collision data with the latest HLT software. [15] 
mixes approaches along with hyper-threading. The threading configurations does not affect the Cost framework, only the recorded Cost results.

\section{SUMMARY AND FUTURE DEVELOPMENTS}

The Cost studies help to validate the HLT software by comparing expected and measured Trigger performance. Based on global mean time per event one can use the maximum input data rate to the Trigger to calculate the number of CPUs needed (in terms of total HEPSPEC benchmark [16] performance), also taking into account processor efficiency when using a farm with mixed processor types.

The Cost Monitoring framework should be validated before the data taking during Run 3 and integrated into the workflow of processing new data. Every day summaries will be produced, therefore the HLT will be well monitored. Also, it needs to be maintained for offline analysis performed by developers until the end of Run 3. Many tests of HLT are performed in order to find the best multi-threading configuration and acquire a good overview of components of the system. Some of the tests are executed on different machines on the HLT computing farm to obtain unbiased performance measurement and compare it to the parameters measured during Run 2.

The TriggerCostBrowser website is maintained and improved. New features, including summary comparisons or more metadata of Trigger execution, are added to ensure a good user experience and high accessibility. The website is also migrated to the new hosting platform.

\section{REFERENCES}

[1] ATLAS Collaboration et al.: The ATLAS Experiment at the CERN Large HadronCollider. Journal of Instrumentation, Vol. 3, 2008, Art. No. S08003, doi: 10.1088/17480221/3/08/S08003.

[2] ATLAS Collaboration: The ATLAS Collaboration Software and Firmware. Report ATL-SOFT-PUB-2021-001, 2021, https://cds.cern.ch/record/2767187

[3] ATLAS Experiment: Approved DAQ Plots. https://twiki.cern.ch/twiki/bin/ view/AtlasPublic/ApprovedPlotsDAQ.

[4] ATLAS Collaboration et al.: Performance of the ATLAS Trigger System in 2015. The European Physical Journal C, Vol. 77, 2017, No. 5, Art. No. 317, doi: 10.1140/epjc/s10052-017-4852-3.

[5] Brun, R.-Rademakers, F.: ROOT: An Object Oriented Data Analysis Framework. In: Werlen, M., Perret-Gallix, D. (Eds.): New Computing Techniques in Physics Research V (AIHENP '96). Nuclear Instruments and Methods in Physics Research Section A: Accelerators, Spectrometers, Detectors and Associated Equipment, Vol. 389, 1997, No. 1-2, pp. 81-86, doi: 10.1016/S0168-9002(97)00048-X 
[6] ATLAS Collaboration: Trigger Monitoring and Rate Predictions Using Enhanced Bias Data from the ATLAS Detector at the LHC. Report ATL-DAQ-PUB-2016-002, https://cds.cern.ch/record/2223498

[7] ATLAS Collaboration: Athena. 2020, doi: 10.5281/zenodo.2641996.

[8] Barrand, G.-Belyaev. I.-Binko, P.-Cattaneo, M.-Chytracek, R.Corti, G.-Frank, M.-Gracia, G.-Harvey, J.-van Herwijnen, E.Maley, P.-Mato, P.-Probst, S.-Ranjard, F.: GAUDI - A Software Architecture and Framework for Building HEP Data Processing Applications. Computer Physics Communications, Vol. 140, 2001, No. 1-2, pp. 45-55, doi: 10.1016/s00104655(01)00254-5.

[9] Rupp, K.: Microprocessor Trend Data. [Online; accessed 30-08-2021] https:// github.com/karlrupp/microprocessor-trend-data.

[10] McCallum, J.: Memory Prices (1957-2018). [Online; accessed 30-08-2021] http: //jcmit.net/memoryprice.htm

[11] Calafiura, P.-Leggett, C.-Seuster, R.-Tsulaia, V.-Van Gemmeren, P.: Running ATLAS Workloads Within Massively Parallel Distributed Applications Using Athena Multi-Process Framework (AthenaMP). Journal of Physics: Conference Series, Vol. 664, 2015, Art. No. 072050, doi: 10.1088/1742-6596/664/7/072050.

[12] Leggett, C.-Baines, J.-Bold, T.-Calafiura, P.-Farrell, S.van Gemmeren, P.-Malon, D.--Ritsch, E.-Stewart, G.-Snyder, S.TsulaiA, V.-Wynne, B.: AthenaMT: Upgrading the ATLAS Software Framework for the Many-Core World with Multi-Threading, Journal of Physics: Conference Series, Vol. 898, 2017, Art. No. 042009, doi: 10.1088/1742-6596/898/4/042009.

[13] Hegner, B.-Mato, P.-Piparo, D.: Evolving LHC Data Processing Frameworks for Efficient Exploitation of New CPU Architectures. 2012 IEEE Nuclear Science Symposium and Medical Imaging Conference Record (NSS/MIC), 2012, pp. 2003-2007, doi: 10.1109/NSSMIC.2012.6551463.

[14] Robison, A. D.: Intel@Threading Building Blocks (TBB). In: Padua, D. (Ed.): Encyclopedia of Parallel Computing. Springer, Boston, 2011, pp. 955-964, doi: 10.1007/978-0-387-09766-4_51.

[15] ATLAS Experiment: Trigger Operation Public Results. https://twiki.cern.ch/ twiki/bin/view/AtlasPublic/TriggerOperationPublicResults.

[16] HEPiX Benchmarking Working Group: HEP-SPEC06 (HS06). https://w3.hepix. org/benchmarking.html. 


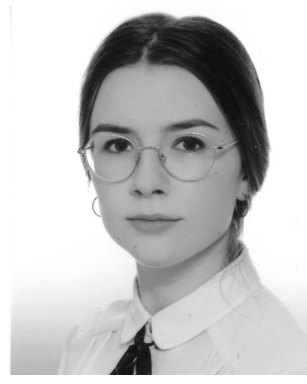

Aleksandra Poreba is part of the ATLAS Collaboration at CERN since March 2019 in HLT core software area. Since January 2020 she works as Trigger Rates and Cost Monitoring Coordinator developing performance monitoring software and preparing studies and estimations of HLT capabilities. She is a fifthyear student of applied computer science at the Faculty of Physics and Applied Computer Science at Akademia Górniczo-Hutnicza, AGH UST in Cracow, Poland with a specialization in modeling and data analysis. The previous year she spent on a Technical Student Programme at CERN. 